\title{
A Parade on Molecular Control of Flowering
}

\author{
Darshan. S. ${ }^{1 *}$, Hemanth ${ }^{2}$, Priya. R.U. ${ }^{3}$
}

\author{
${ }^{1,3}$ Ph.D. Scholars, College of Agriculture, Vellayani, Kerala Agricultural University, Kerala, India \\ ${ }^{2} \mathrm{Ph}$.D. Scholar, Institute of Agri-Biotechnology, University of Agricultural Sciences, Dharwad, Karnataka, India
}

\begin{abstract}
Flower is a reproductive part of plant which contains complex array of functionally specialized structures.Photoperiodism, or the ability of an organism to detect day length, makes it possible for an event to occur at a particular time of year, thus allowing for a seasonal response. Circadian rhythms and photoperiodism have the common property of responding to cycles of light and darkness. Plant physiologists believed that the correlation between long days and flowering was a consequence of the accumulation of photosynthetic products synthesized during long days. Vernalization causes stable changes in the pattern of gene expression in the meristem, gene expression that are still stable even after vernalization is removed. The organ identity genes initially were identified through mutations that dramatically alter the structure and thus the identity of the floral organs produced in two adjacent whorls. The patterns of organ formation in the wild type and most of the mutant phenotypes are predicted and explained by ABC model of flower development. the transition to flowering involves a complex system of interacting factors that include among carbohydrates, gibberellins, cytokinins, bromeliads and ethylene.
\end{abstract}

Keywords-Flower, Floral evocation Vernalization, ABC model.

\section{INTRODUCTION}

Flower is a reproductive part of plant which contains complex array of functionally specialized structures, which is often brightly colored, it eventually develops into a seed or fruit. It contains four distinct structures or floral organs viz.,Calyx (Sepals), Corolla (Petals), Stamens (Pollens) and Carpels (ovaries) These floral organs are located on concentric rings which are called as whorls. The radical chain of events which commit the shoot apical meristem to produce flowers is collectively referred to as floral evocation. The developmental signals that bring about floral evocation include, Internal cues - Circadian rhythms (Bünning1960), phase change (Clark 1983), genotype and hormones. External cues - Day length (photoperiod) and temperature (vernalization) (Lang 1965).

The interactions of these endogenous and external factors enable plants to synchronize their reproductive development with the environment.Based on the developmental signal the regulation of flowering is categorized into three types viz.,
1. Autonomous regulation- The type of flowering which occurs strictly in response to internal developmental factors and does not depend on any particular environmental conditions is referred to as autonomous regulation.

2. Obligate or Qualitative regulation- The type of flowering which involves an absolute requirement for the proper environmental cues.

3. Facultative regulation- The type of flowering which is promoted by certain environmental cues but will eventually occur in the absence of such cues.

\section{INTERNAL CUES - FLORAL EVOCATION PHASE CHANGE}

In humans we have different stages like infancy, childhood, adolescence, and adulthood. For us puberty is the dividing line between the non-reproductive and the reproductive phases. Similarly all multicellular organisms pass through a series of more or less defined developmental stages, each with its characteristic features. In animals developmental changes take place throughout the entire organism. In higher plants developmental changes occur in a single, dynamic region i.e. in shoot apical meristem. Shoot apical meristems has three developmental phases viz.Juvenile phase, Adult vegetative phase and Adult reproductive phase. This transition from one phase to another is called phase change.Among them the transition from juvenile phase to adult vegetative phase is gradual and slow, whereas the transition from adult vegetative phase to adult reproductive phase is abrupt and fast.

This phase change can be influenced by nutrients and hormones. In many plants, exposure to low-light conditions prolongs juvenility or causes reversion to juvenility. A major consequence of the low-light regime is a reduction in the supply of carbohydrates to the apex which reduces the size of the apex; thus carbohydrate supply (especially sucrose) plays a major role in the transition between juvenility and adult. e.g. Chrysanthemum, flowers are not initiated until a minimum apex size has been reached. Experimental evidence shows that the application of gibberellins causes reproductive structures to form in young, juvenile plants of several conifer families. Although gibberellins promote the 
attainment of reproductive maturity in conifers and many herbaceous angiosperms as well, GA3 causes rejuvenation in Hederaand in several other woody angiosperms.

The term juvenility has different meanings for herbaceous and woody species. Juvenile herbaceous meristems flower readily when grafted onto flowering adult plants whereas Juvenile woody meristems generally do not. Extensive studies in tobacco have demonstrated that floral evocation requires the apical bud to pass through two developmental stages for it to flower viz. Competent and Determined (McDaniel et al. 1992). A bud is said to be competentif it is able to flower when given the appropriate developmental signal. For example, if a vegetative shoot (scion) is grafted onto a flowering stock and the scion flowers immediately, then the scion is said to be competent.A bud is said to be determined if it progresses into flowering even after being removed from its normal context i.e. a florally determined bud will produce flowers even if it is grafted onto a vegetative plant that is not producing any floral stimulus.

\section{CIRCADIAN RHYTHMS}

Circadian rhythm is derived from the Latin term circa diemmeaning "approximately a day". It refers to the rhythmic changes in the organisms which cycle alternatively through high-activity and low-activity phases with a regular periodicity of 24 hours. Examples- Leaf and petal movements (day and night positions), Stomatal opening and closing, Growth and sporulation patterns in fungi (e.g., Pilobolus), Pupal emergence(the fruit fly Drosophila), Activity cycles in rodents, Photosynthetic capacity and respiration rate. Circadian rhythms cannot be the direct responses to the presence or absence of light but must be based on an internal pacemaker, often called an endogenous oscillator, which is coupled to a variety of physiological processes such as leaf movement or photosynthesis and it maintains the rhythm.

Circadian rhythms arise from cyclic phenomena that are defined by three parameters viz. period, phase and amplitude. Period is time between comparable points in the repeating cycle, typically the period is measured as the time between consecutive maxima (peaks) or minima (troughs). Phase is regarded as any point in the cycle that is recognizable by its relationship to the rest of the cycle, the most obvious phase points are the peak and trough position.Amplitude is usually considered to be the distance between peak and trough, the amplitude of a biological rhythm can often vary while the period remains unchanged.Ideally the endogenous oscillator is entrained (synchronized) to a true 24-hour period by environmental signals, the most important of which are dusk and dawn. Such environmental signals are termed zeitgebers (German for "time givers"). When zeitgebers are removed rhythm is said to be free-runningi.e. it reverts to the circadian period that is characteristic of the particular organism. In free running, the rhythms depart from an exact 24-hour period, which then drift in relation to solar time either gaining or losing time depending on whether the period is shorter or longer than 24 hours. Many rhythms damp out (i.e., the amplitude decreases). Any type ofzeitgebercan restart the rhythm.

In circadian rhythms, the operation of the endogenous oscillator sets a response to occur at a particular time of day. Investigators test the response of the endogenous oscillator by placing the organism in continuous darkness and examining the response to a short pulse of light (usually less than 1 hour) given at different phase points in the free-running rhythm. (Subjective day, it is said to be the phase of the rhythm that coincides with the light period of the previous entraining cycle and the phase that coincides with the dark period is called the subjective night) When the light pulse is given in first few hours of the subjective night, the rhythm is delayed because the organism interprets the light pulse as the end of the previous. When the light pulse is given in the end of the subjective night, phase of the rhythm is advanced because now the organism interprets the light pulse as the beginning of the following day.

\section{EXTERNAL CUES - FLORAL EVOCATION PHOTOPERIODISM}

Photoperiodism, or the ability of an organism to detect day length, makes it possible for an event to occur at a particular time of year, thus allowing for a seasonal response. Circadian rhythms and photoperiodism have the common property of responding to cycles of light and darkness.

Precisely at the equator, day length and night length are equal and constant throughout the year. As one moves away from the equator toward the poles, the days become longer in summer and shorter in winter makes it possible for an event to occur at a particular time of year, thus allowing for a seasonal response. Plant species have evolved to detect these seasonal changes in day length, and their specific photoperiodic responses are strongly influenced by the latitude from which they originated.

Responses controlled by day length are numerous. In the animal, day length controls seasonal activities as hibernation, development of summer or winter coats, and reproductive activity. In plants initiation of flowering, asexual reproduction, the formation of storage organs, and the onset of dormancy.Numerous plant species flower during the long days of summer. Plant physiologists believed that the correlation between long days and flowering was a consequence of the accumulation of photosynthetic products synthesized during long days. This Hypothesis was disproved by Wightman Garner and 
Henry Allard (1920s) U.S. Department of Agriculture laboratories in Beltsville, Maryland. Mutant variety of tobacco, Maryland Mammoth, grew profusely to about $5 \mathrm{~m}$ in height but failed to flower in the prevailing conditions of summer. However, the plants flowered in the greenhouse during winter under natural light conditions. The two main photoperiodic response categories are shortday plants and long-day plants. Short-day plants (SDPs) flower only in short days (qualitative SDPs), or their flowering is accelerated by short days (quantitative SDPs) and Long-day plants (LDPs) flower only in long days (qualitative LDPs), or their flowering is accelerated by long days (quantitative LDPs). The distinction between long-day and short-day plants is that flowering in LDPs is promoted only when the day length exceeds a certain duration, called the critical day length, in every 24-hour cycle, whereas promotion of flowering in SDPs requires a day length that is less than the critical day length.

\section{VERNALIZATION}

Vernalizationis the process whereby flowering is promoted by a cold treatment $\left(1\right.$ to $\left.7^{\circ} \mathrm{C}\right)$ given to a fully hydrated seed or to a growing plant. Dry seeds do not respond to the cold treatment. Without the cold treatment, plants that require vernalization show delayed flowering or remain vegetative. In many cases these plants grow as rosettes with no elongation of the stem.

Vernalization causes stable changes in the pattern of gene expression in the meristem, gene expression that are still stable even after vernalization is removed. This is an example of epigenetic regulation. This has been confirmed in winter-annual ecotypes of Arabidopsis that require both vernalization and long days to flower, a gene that acts as a repressor of flowering is FLOWERING LOCUS C (FLC). $F L C$ is highly expressed in non-vernalized shoot apical meristems after vernalization, this gene is epigenetically switched off by an unknown mechanism for the remainder of the plant's life cycle, permitting flowering in response to long days to occur. In the next generation, however, the gene is switched on again restoring the requirement for cold. Thus in Arabidopsis, the state of expression of the FLC gene represents a major determinant of meristem competence (Michaels and Amasino 2000).

\section{PHYSIOLOGY OF FLOWERING}

Floral meristems usually can be distinguished from vegetative meristems, even in the early stages of reproductive development, by their larger size. The transition from vegetative to reproductive development is marked by an increase in the frequency of cell divisions within the central zone of the shoot apical meristem. In the vegetative meristem, the cells of the central zone complete their division cycles slowly. As reproductive development commences, the increase in the size of the meristem is largely a result of the increased division rate of these central cells. Recently, genetic and molecular studies have identified a network of genes that control floral morphogenesis in Arabidopsis, snapdragon (Antirrhinum), and other species.

\section{FLORAL DEVELOPMENT IN ARABIDOPSIS}

During the vegetative phase of growth, the Arabidopsis vegetative apical meristem produces phytomeres with very short internodes, resulting in a basal rosette of leaves (Phytomereconsists of a leaf, the node to which the leaf is attached, theaxillary bud, and the internode below the node).As plants initiate reproductive development, the vegetativemeristem is transformed into an indeterminate primaryinflorescence meristem that produces floral meristemson its flanks. The lateral buds of the cauline leaves (inflorescence leaves) develop into secondaryinflorescence meristems, and their activity repeatsthe pattern of development of the primary inflorescencemeristem. (Bewley et al. 2000)

Floral meristems initiate four different types of floral organs: sepals, petals, stamens, and carpels (Coen and Carpenter 1993). These sets of organs are initiated in concentric rings, called whorls, around the flanks of the meristem .The initiation of the innermost organs, the carpels, consumes all of the meristematic cells in the apical dome, and only the floral organ primordia are present as the floral bud develops. In the wild-type Arabidopsis flower, the whorls are arranged as follows:

- The first (outermost) whorl consists of four sepals, which are green at maturity.

- The second whorl is composed of four petals, which are white at maturity.

- The third whorl contains six stamens, two of which are shorter than the other four.

- The fourth whorl is a single complex organ, the gynoecium or pistil, which is composed of an ovary with two fused carpels, each containing numerous ovules, and a short style capped with a stigma.

Mutations have identified three classes of genes that regulate floral development: floral organ identity genes, cadastral genes, and meristem identity genes.

1. Floral organ identity genes directly control floral identity. The proteins encoded by these genes are transcription factors that likely control the expression of other genes whose products are involved in the formation and/or function of floral organs.

2. Cadastral genes act as spatial regulators of the floral organ identity genes by setting boundaries for their expression. (The word cadastre refers to a map or survey showing property boundaries for taxation purposes.)

3. Meristem identity genes are necessary for the initial induction of the organ identity genes. 
These genes are the positive regulators of floral organ identity.

\section{ACTIVITY OF MERISTEM IDENTITY GENES}

Meristem identity genes must be active for the primordial formed at the flanks of the apical meristem to become floral meristems. For example, mutants of Antirrhinum (snapdragon) that have a defect in the meristem identity gene FLORICAULA develop an inflorescence that does not produce flowers. Instead of causing floral meristems to form in the axils of the bracts, the mutant floricaula gene results in the development of additional inflorescence meristems at the bract axils. The wild-type floricaula (FLO) gene controls the determination step in which floral meristem identity is established. In Arabidopsis, AGAMOUS-LIKE 201 (AGL20), APETALA1 (AP1), and LEAFY (LFY) are all critical genes in the genetic pathway that must be activated to establish floral meristem identity. LFY is the Arabidopsis version of the snapdragon FLO gene. AGL20 plays a central role in floral evocation by integrating signals from several different pathways involving both environmental and internal cues (Borner et al. 2000). AGL20 thus appears to serve as a master switch initiating floral development. Once activated, AGL20 triggers the expression of LFY, and LFY turns on the expression of AP1 (Simon et al. 1996). In Arabidopsis, LFY and AP1 are involved in a positive feedback loop; that is, AP1 expression also stimulates the expression of LFY.

\section{ORGAN IDENTITY GENES}

The genes that determine floral organ identity were discovered as floral homeotic mutants mutations in the fruit fly(Drosophila) led to the identification of a set of homeotic genes encoding transcription factors that determine the locations at which specific structures develop. Such genes act as major developmental switches that activate the entire genetic program for a particular structure. The expression of homeotic genes thus gives organs their identity.

Dicot flowers consist of successive whorls of organs that form as a result of the activity of floral meristems: sepals, petals, stamens, and carpels. These organs are produced when and where they are because of the orderly, patterned expression and interactions of a small group of homeotic genes that specify floral organ identity.

The floral organ identity genes were identified through homeotic mutations that altered floral organ identity so that some of the floral organs appeared in the wrong place. For example, Arabidopsis plants with mutations in the APETALA2 (AP2) gene produce flowers with carpels where sepals should be, and stamens where petals normally appear. The homeotic genes that have been cloned so far encode transcription factors (proteins that control the expression of other genes). Most plant homeotic genes belong to a class of related sequences known as MADS box genes, whereas animal homeotic genes contain sequences called homeoboxes.Many of the genes that determine floral organ identity are MADS box genes, including the DEFICIENS gene of snapdragon and the AGAMOUS, PISTILLATA1, and APETALA3 genes of Arabidopsis. The MADS box genes share a characteristic, conserved nucleotide sequence known as a MADS box, which encodes a protein structure known as the MADS domain. The MADS domain enables these transcription factors to bind to DNA that has a specific nucleotide sequence.Not all genes containing the MADS box domain are homeotic genes. For example, AGL20 is a MADS box gene, but it functions as a meristem identity gene.

\section{Three types of homeotic genes control floral organ identity}

Five different genes are known to specify floral organ identity in Arabidopsis: APETALA1 (AP1), APETALA2 (AP2), APETALA3 (AP3), PISTILLATA (PI), and AGAMOUS (AG) (Bowman et al. 1989; Weigel and Meyerowitz 1994). The organ identity genes initially were identified through mutations that dramatically alter the structure and thus the identity of the floral organs produced in two adjacent whorls. For example, plants with the ap 2 mutation lack sepals and petals. Plants bearing ap3 or $p i$ mutations produce sepals instead of petals in the second whorl, and carpels instead of stamens in the third whorl. And plants homozygous for the agmutation lack both stamens and carpels.

Because mutations in these genes change floral organ identity without affecting the initiation of flowers, they are homeotic genes. These homeotic genes fall into three classes viz., Types A, B, and C defining three different kinds of activities.

1. Type A activity, encoded by AP1 and AP2, controls organ identity in the first and second whorls. Loss of type A activity results in the formation of carpels instead of sepals in the first whorl, and of stamens instead of petals in the second whorl.

2. Type B activity, encoded by AP3 and PI, controls organ determination in the second and third whorls. Loss of type B activity results in the formation of sepals instead of petals in the second whorl, and of carpels instead of stamens in the third whorl.

3. Type C activity, encoded by AG, controls events in the third and fourth whorls. Loss of type $\mathrm{C}$ activity results in the formation of petals instead of stamensin the third whorl, and replacement of the fourth whorl by a new flower such that the 
fourth whorl of the ag mutant flower is occupied by sepals.

The role of the organ identity genes in floral development is dramatically illustrated by experiments in which two or three activities are eliminated by loss-offunction mutations. Quadruple-mutant plants (ap1, ap2, $a p 3 / p i$, and $a g$ ) produce floral meristems that develop as pseudoflowers; all the floral organs are replaced with green leaf like structures, although these organs are produced with a whorledphyllotaxy. Evolutionary biologists, beginning with the eighteenth century German poet, philosopher, and natural scientist Johann Wolfgang von Goethe (1749-1832), have speculated that floral organs are highly modified leaves, and this experiment gives direct support to these ideas.

\section{ABC MODEL}

In 1991 the ABC model was proposed by George Haughn and Chris Somerville to explain how homeotic genes control organ identity. The ABC model postulates that organ identity in each whorl is determined by a unique combination of the three organ identity gene activities.

- Activity of type A alone specifies sepals.

- Activities of both A and B are required for the formation of petals.

- Activities of B and C form stamens.

- Activity of $\mathrm{C}$ alone specifies carpels.

The model further proposes that activities $\mathrm{A}$ and $\mathrm{C}$ mutually repress each other; that is, both $\mathrm{A}$ and C-type genes have cadastral function in addition to their function in determining organ identity.

The patterns of organ formation in the wild type and most of the mutant phenotypes are predicted and explained by this model. The challenge now is to understand how the expression pattern of these organ identity genes is controlled by cadastral genes; how organ identity genes, which encode transcription factors, alter the pattern of other genes expressed in the developing organ; and finally how this altered pattern of gene expression results in the development of a specific floral organ.

\section{MULTIPLE FACTORS AND PATHWAYS TO FLOWER}

It is clear that the transition to flowering involves a complex system of interacting factors that include, among others, carbohydrates, gibberellins, cytokinins, and, in the bromeliads, ethylene. Leaf-generated transmissible signals are required for determination of the shoot apex in both autonomously regulated and photoperiodic species. Determining whether these transmissible signals consist of single or multiple components is a major challenge for the future.

Recent genetic studies have established that there arefour genetically distinct developmental pathways that control flowering in the LDP Arabidopsis. (Blazquez 2000)
1. The photoperiodic pathway involves phyto chromes and crypto chromes. The interaction of these photoreceptors with acircadian clock initiates a pathway that eventually results in the expression of the gene CONSTANS(CO), which encodes a zincfinger transcription factorthat promotes flowering. $\mathrm{CO}$ acts through other genes to increase the expression of the floral meristem identity gene LEAFY (LFY).

2. In the dual autonomous/vernalization pathway, flowering occurs either in response to internal signals (theproduction of a fixed number of leaves) or to low temperatures. In the autonomous pathway of Arabidopsis, all of the genes associated with the pathway are expressed in the meristem. The autonomous pathway acts by reducing the expression of the flowering repressor gene FLOWERING LOCUS C (FLC), an inhibitor of LFY (Michaels and Amasino 2000).Vernalization also represses FLC, but perhaps by a different mechanism (an epigenetic switch). Becausethe FLC gene is a common target, the autonomous and vernalization pathways are grouped together.

3. The carbohydrate, or sucrose, pathway reflects the metabolicstate of the plant. Sucrose stimulates floweringin Arabidopsis by increasing LFY expression, although the genetic pathway is unknown.

4. The gibberellin pathway is required for early flowering and for flowering under non-inductive short days.

All four pathways converge by increasing the expression of the key floral meristem identity gene AGAMOUSLIKE20 (AGL20). The role of AGL20, a MADS box-containing transcription factor, is to integrate the signals coming from all four pathways into a unitary output. Obviously the strongest output signal occurs when all four pathways are activated.

Although many pathways feed into AGL20, there mustbe some redundancy in the system because flowering isonly delayed, but not completely blocked, in ag120 mutants. Thus, one or two other genes must be able to take over the role of AGL20 when it is mutated. Once turned on by AGL20, LFY activates the floral homoerotic genes [(APETALA1 (AP1), APETALA3 (AP3),PISTILLATA (PI), and AGAMOUS (AG)] that are required for floral organ development. APETALA2 (AP2) is expressed in both vegetative and floral meristems and is therefore not affected by LFY. However, AP2 exerts a negative effect on AG expression.

Besides serving as a floral homeotic gene, AP1 functions as a meristem identity gene in Arabidopsis because it is involved in a positive feedback loop with LFY. 
Consequently, once the transition to flowering has reached this stage, flowering is irreversible. The existence of multiple flowering pathways provides angiosperms with maximum reproductive flexibility, enabling them to produce seeds under a wide variety of conditions. Redundancy within the pathways ensures that reproduction, the most crucial of all physiological functions, will be relatively insensitive to mutations and evolutionarily robust.

\section{CONCLUSION}

Flowering is a complex process which involves special gene types which recognize the internal and external cues and select the ideal conditions to flower. Flowering was first studied in Arabidopsis which helped in identifying flowering genes namely floral meristem identity genes, organ identity genes and Cadastral genes. Among them organ identity genes is further classified into type A, type $\mathrm{B}$ and type $\mathrm{C}$ genes.

\section{REFERENCES}

[1] Bewley, J. D., Hempel, F. D., McCormick, S. and Zambryski, P. 2000. Reproductive Development. In: Biochemistry and Molecular Biology of Plants, B. B. Buchanan, W. Gruissem, and R. L. Jones (eds.), American Society of Plant Biologists, Rockville, MD.

[2] Blazquez, M. A. 2000. Flower development pathways. $J$. Cell Sci. 113: 3547-3548.

[3] Blazquez, M. A. and Weigel, D. 2000.Integration of floral inductive signals in Arabidopsis. Nature 404: 889892.

[4] Borner, R., Kampmann, G., Chandler, J., Gleissner, R., Wisman, E., Apel, K. and Melzer, S. 2000. A MADS domain gene involved in the transition to flowering in Arabidopsis. Plant J. 24: 591-599.

[5] Bowman, J. L., Smyth, D. R. and Meyerowitz, E. M. 1989. Genes directing flower development in Arabidopsis. Plant Cell 1: 37-52.

[6] Bünning, E. 1960.Biological clocks.Cold Spring HarborSymp.Quant.Biol. 15: 1-9.

[7] Clark, J. R. 1983. Age-related changes in trees. J. Arboriculture 9: 201-205.

[8] Coen, E. S. and Carpenter, R. 1993.The metamorphosis of flowers.Plant Cell 5: 1175-1181.

[9] Devlin, P. F. and Kay, S. A. 2000.Cryptochromes are required for phytochromesignaling to the circadian clock but not for rhythmicity. Plant Cell 12: 2499-2509.

[10]Gasser, C. S. and Robinson-Beers, K. 1993.Pistil development.Plant Cell 5: 1231-1239.

[11] Guo, H., Yang, H., Mockler, T. C. and Lin, C. 1998. Regulation of flowering time by Arabidopsis photoreceptors. Science 279:1360-1363.
[12]Lang, A. 1965.Physiology of flower initiation. In Encyclopedia of Plant Physiology (Old Series, Vol. 15), W. Ruhland, (ed.), Springer, Berlin, pp. 1380-1535.

[13]Lang, A., Chailakhyan, M. K. and Frolova, I. A. 1977. Promotion and inhibition of flower formation in a dayneutral plant in grafts with a short-day plant and a long-day plant. Proc. Natl. Acad. Sci. USA 74: 24122416.

[14] McDaniel, C. N., Singer, S. R. and Smith, S. M. E. 1992. Developmental states associated with the floral transition. Dev. Biol. 153: 59-69.

[15] Michaels, S. D. and Amasino, R. M. 2000. Memories of winter: Vernalization and the competence to flower. Plant Cell Environ. 23: 1145-1154.

[16] Papenfuss, H. D. and Salisbury, F. B. 1967.Aspects of clock resetting in flowering of Xanthium.Plant Physiol. 42: 1562-1568.

[17] Purvis, O. N. and Gregory, F. G. 1952.Studies in vernalization of cereals. XII. The reversibility by high temperature of the vernalized condition in Petkus winter rye. Ann. Bot. 1: 569-592.

[18] Salisbury, F. B. 1963. Biological timing and hormone synthesis in flowering of Xanthium.Planta49: 518-524.

[19] Simon, R., Igeno, M. I. and Coupland, G. 1996. Activation of floral meristem identity genes in Arabidopsis.Nature 384: 59-62.

[20] Taiz, L. and Zeiger, E. 2006.Plantphysiology $5^{\text {th }} \mathrm{Ed}$ Sinauer Associates. 764p.

[21] Vince-Prue, D. 1975.Photoperiodism in Plants.McGrawHill, London.

[22] Weigel, D. and Meyerowitz, E. M. 1994.The ABCs of floral homeotic genes.Cell 78: 203-209.

[23] Wilson, R. A., Heckman, J. W. and Sommerville, C. R. 1992. Gibberellin is required for flowering in Arabidopsis thaliana under short days. Plant Physiol. 100: 403-408. 